PATIENT SAFETY

\author{
S. Kakeda \\ Y. Korogi \\ M. Ogawa \\ K. Otsubo \\ Y. Morishita
}

\section{Reduction of the Radiation Dose for Multidetector Row CT Angiography of Cerebral Aneurysms Using an Edge-Preserving Adaptive Filter: A Vascular Phantom Study}

SUMMARY: To determine how much the radiation dose can be reduced in multidetector row CTA using a QDS, we performed CTA at various exposure settings using a vascular phantom simulating various aneurysms with superimposed bone skull structures, and postprocessed the image data with QDS. Our results demonstrated that the radiation dose of CTA can be reduced by at least $25 \%$ and the image quality for visualizing aneurysms can be preserved by applying the ODS.

\begin{abstract}
ABBREVIATIONS: CNR = contrast-to-noise ratio; $\mathrm{CTA}=\mathrm{CT}$ angiography; $\mathrm{CTDIw}=$ weighted $\mathrm{CT}$ dose index; MTF = modulation transfer function; Q04 = mild QDS; Q06 = heavy sharpening QDS; $\mathrm{Q} 08$ = heavy smoothing QDS; QDS = quantum denoising system; ROI = region of interest; $\mathrm{W}=$ weight of blending the smoothed image and the sharpened image
\end{abstract}

A lthough CTA is an efficient noninvasive imaging method for the evaluation of intracranial aneurysms, there is a trend toward using an increased radiation dose with multidetector row CT in comparison with the dose used for singledetector row CT. Many investigators recommend low-tubevoltage scanning with properly adjusted tube current for reducing the radiation dose during cerebral CT because a photoelectric effect in $\mathrm{x}$-ray attenuation increases at lower tube voltages. ${ }^{1}$ On the other hand, reducing the radiation dose causes an increase in the image noise content. Currently, several previous studies have reported that the edge-preserving adaptive filter decreases qualitative and quantitative noise in low-dose CT images. ${ }^{2}$ However, the effect of this filter on the lesion conspicuity of cerebral CTA has not been investigated. Recently, a new edge-preserving adaptive filter known as the QDS (Toshiba Medical Systems, Tokyo, Japan) has been developed, which can selectively eliminate the noise without affecting the resolution in low-dose scanning. The purpose of this phantom study was to assess how much the radiation dose in CTA for cerebral aneurysms can be reduced by using the QDS.

\section{Technique}

\section{Phantom Design}

An anthropomorphic vascular phantom made of silicone rubber with superimposed bone skull structures was designed to simulate bilateral intracranial arteries with various intracranial aneurysms. Two types of simulated aneurysms ( 10 aneurysms with diameters of $3 \mathrm{~mm}$ and 6 aneurysms with diameters of $6 \mathrm{~mm}$ ) were placed on the simulated intracranial artery. Six aneurysms with diameters of $6 \mathrm{~mm}$ had an

Received February 12, 2009; accepted after revision June 30.

From the Department of Radiology (S.K., Y.K., M.O.), University of Occupational and Environmental Health School of Medicine, Kitakyushu, Japan; and Medical Systems Division (K.O., Y.M.), Toshiba Corporation, Tokyo, Japan.

Please address correspondence to Shingo Kakeda, MD, Department of Radiology, University of Occupational and Environmental Health, 1-1 Iseigaoka, Yahatanishi-ku, Kitakyushu 807-8555, Japan; e-mail: kakeda@med.uoeh-u.ac.jp

DOI 10.3174/ajnr.A1798 aneurysmal bleb with a diameter of $2 \mathrm{~mm}$. This phantom was filled with 2 kinds of contrast material concentrations (20 and $15 \mathrm{mg} \mathrm{I} / \mathrm{mL}$ ).

\section{Image Acquisition}

CTA was performed with 3 tube voltages in combination with various effective tube-current settings by using a 64-section scanner (Aquilion 64, Toshiba Medical Systems) (Table). For all CTA, we kept constant the following parameters: detector configuration of $64 \times 0.5$ $\mathrm{mm}$, section thickness of $0.5 \mathrm{~mm}$, reconstruction interval of $0.3 \mathrm{~mm}$, beam pitch of 1.5:1, and table speed of $15 \mathrm{~mm}$ per gantry rotation (0.8-second gantry rotation time). CTA is routinely performed at 120 $\mathrm{kVp}$ and $200 \mathrm{mAs}$. Therefore, CTA without QDS at $120 \mathrm{kVp}$ and 200 $\mathrm{mAs}$ was defined as the standard CTA.

\section{Filter Processing}

The algorithms used in the QDS are shown in simplified form in Fig 1. ${ }^{3}$ For edge-detection processing, the edge elements that should be maintained are extracted from the input image by using the edgedetection filter. The extracted edge elements are used to calculate a suitable blending ratio $(0 \leq \mathrm{w} \leq 1)$ for the smoothed image and the sharpened image on the basis of the edge-sensitivity curve. Smoothing processing (low-pass filtering) was performed on the input image by using the smoothing filter to attenuate noise elements, while sharpening processing (high-pass filtering) was performed on the input image by using the sharpening filter to enhance the fine structures. The QDS reduces noise by increasing the blending ratio of the smoothed image in areas with low edge intensity, while maintaining the resolution in regions near the edges by increasing the blending ratio of the sharpened image in areas with high edge intensity. The edge-sensitivity curve was used to adjust the sensitivity for edges (the degree to which the edges are maintained) when blending the smoothed image and the sharpened image. Each type of filter processing was in 3D.

According to the coding of the manufacturer, the filters were classified as Q04, Q06, and Q08. The Q06 and Q08 are more aggressive than the Q04 in terms of the level of both smoothing and sharpening processing. The Q06 and Q08 use the different blending ratios of the smoothed and sharpened image. In comparison with Q06, the Q08 uses a higher ratio of the smoothed image and a lower ratio of the 


\begin{tabular}{|c|c|c|c|c|c|c|c|c|c|c|}
\hline \multicolumn{11}{|c|}{ Mean objective measurements of CNR and mean qualitative image scores ${ }^{a}$} \\
\hline \multirow[b]{3}{*}{ kV } & \multirow[b]{3}{*}{ mAs } & \multirow[b]{3}{*}{ CTDIw } & \multicolumn{4}{|c|}{ CNR } & \multicolumn{4}{|c|}{ Image Quality Score } \\
\hline & & & \multicolumn{2}{|c|}{$15 \mathrm{mg} \mathrm{l} / \mathrm{mL}$} & \multicolumn{2}{|c|}{$20 \mathrm{mg} \mathrm{l} / \mathrm{mL}$} & \multicolumn{2}{|c|}{$15 \mathrm{mg} \mathrm{l} / \mathrm{mL}$} & \multicolumn{2}{|c|}{$20 \mathrm{mg} \mathrm{l} / \mathrm{mL}$} \\
\hline & & & Original & 008 & Original & 008 & Original & 008 & Original & 008 \\
\hline \multirow[t]{3}{*}{100} & 150 & 23.9 & $9.2 \pm 0.6$ & $11.6 \pm 1.0$ & $11.8 \pm 0.9$ & $16.1 \pm 2.5$ & $2.00 \pm 0.7$ & $2.58 \pm 0.8$ & $2.25 \pm 0.8$ & $2.92 \pm 0.8$ \\
\hline & 200 & 31.8 & $10.9 \pm 1.7$ & $14.3 \pm 2.8^{b}$ & $13.8 \pm 2.2$ & $19.2 \pm 3.6$ & $2.17 \pm 0.7$ & $2.50 \pm 1.0$ & $3.17 \pm 0.8$ & $3.58 \pm 0.7^{b}$ \\
\hline & 250 & 39.8 & $12.0 \pm 2.1$ & $16.1 \pm 2.6^{b}$ & $16.0 \pm 1.0$ & $21.8 \pm 1.4^{b}$ & $2.58 \pm 0.7$ & $3.00 \pm 0.4^{b}$ & $3.42 \pm 0.5$ & $3.92 \pm 0.7^{b}$ \\
\hline \multirow[t]{3}{*}{120} & 100 & 24.0 & $8.7 \pm 1.4$ & $11.3 \pm 0.7$ & $11.3 \pm 1.1$ & $14.9 \pm 1.6$ & $1.83 \pm 0.7$ & $2.40 \pm 0.5$ & $2.17 \pm 0.9$ & $2.42 \pm 1.0$ \\
\hline & 150 & 36.0 & $10.2 \pm 1.0$ & $14.2 \pm 1.4^{b}$ & $14.8 \pm 1.2$ & $21.8 \pm 1.8^{b}$ & $2.33 \pm 0.8$ & $2.75 \pm 0.6$ & $2.83 \pm 0.7$ & $3.50 \pm 0.5^{b}$ \\
\hline & 200 & 48.0 & $11.5 \pm 2.0^{c}$ & $18.4 \pm 2.3^{b}$ & $17.3 \pm 1.7^{c}$ & $22.7 \pm 2.3^{b}$ & $2.50 \pm 1.1^{c}$ & $3.08 \pm 0.8^{b}$ & $3.12 \pm 0.7^{c}$ & $3.83 \pm 0.8^{b}$ \\
\hline 135 & 100 & 30.7 & $9.80 \pm 1.6$ & $13.3 \pm 0.9$ & $12.3 \pm 1.9$ & $16.5 \pm 1.6$ & $1.67 \pm 0.7$ & $2.00 \pm 0.7$ & $2.58 \pm 1.0$ & $3.00 \pm 1.0$ \\
\hline
\end{tabular}

a Data are the mean \pm SD

${ }^{b}$ CTA with QDS were significantly superior to the standard CTA (120 kVp and $200 \mathrm{mAs}$ ) with a $P$ value $<.05$ (Wilcoxon signed rank test)

c Standard CTA.

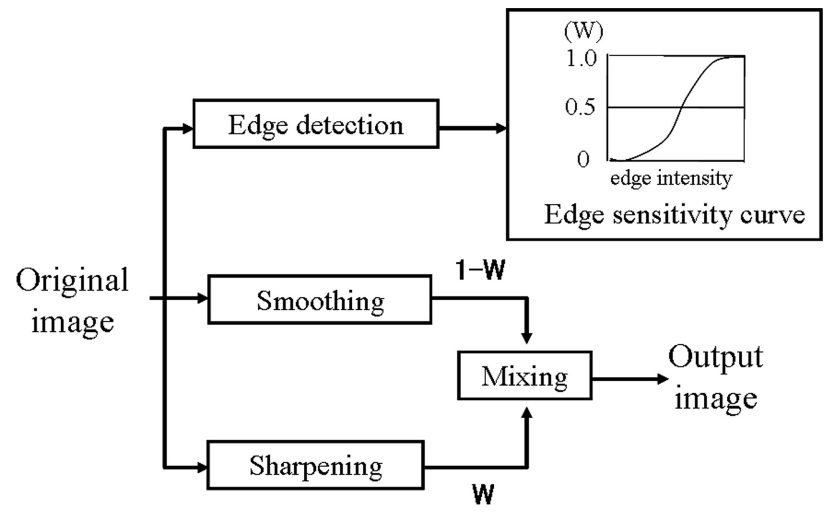

Fig 1. Flow chart depicts the basic processing steps of QDS used in the present study.

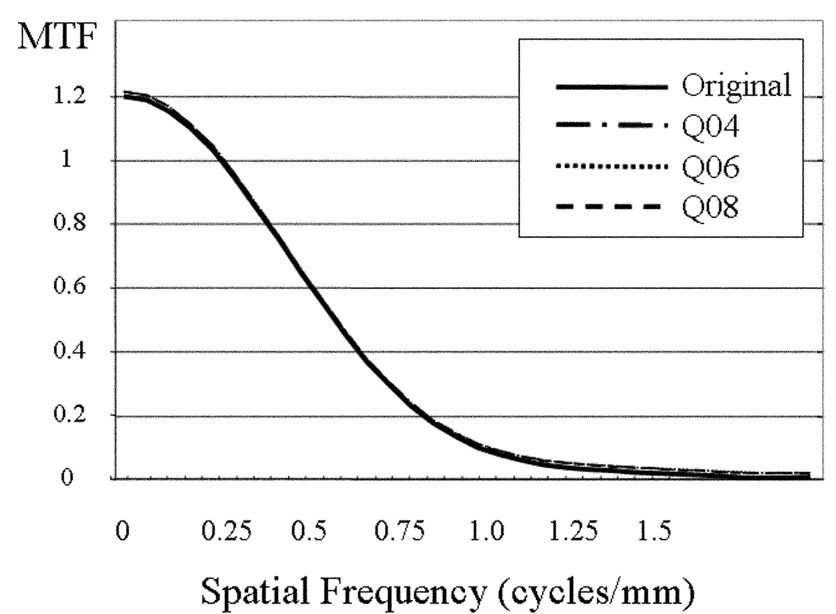

Fig 2. MTF with a line-wire phantom for the original image and 3 ODS-processed images.

sharpened image. CTA was postprocessed with these 3 types of QDSs. Figures 2 and 3 show the MTF and Wiener noise power spectra. There was no meaningful difference in the MTF among the 4 subsets of CT images (Fig 2). The Wiener noise power spectra suggested that Q08 was the most effective in reducing image noise at all spatial frequencies, especially at lower spatial frequencies (Fig 3).

\section{Image Analysis}

The original image and Q08-processed CTA were evaluated. For calculation of the CNR of the aneurysms with respect to the backgrounds, the mean CT number (attenuation) and SD (image noise) of 6 different aneurysms with a diameter of $6 \mathrm{~mm}$ in the vascular phan-

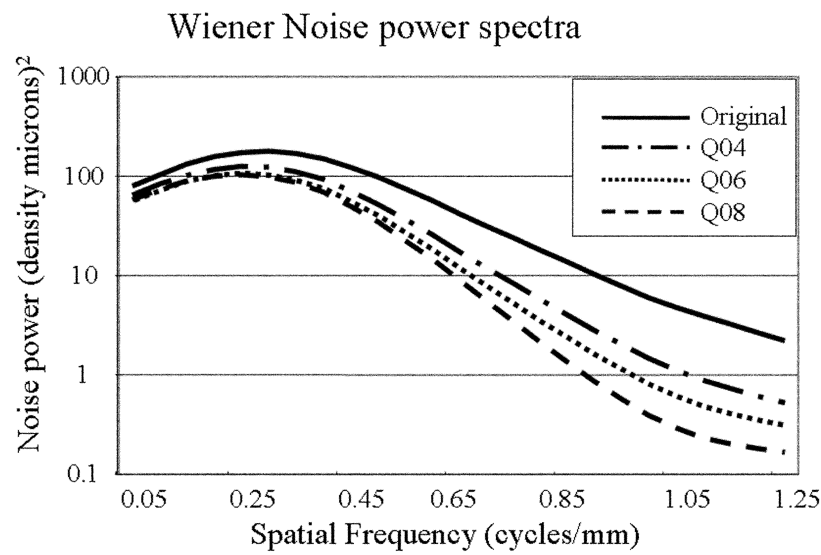

Fig 3. Wiener spectra with a line-wire phantom for the original image and 3 ODSprocessed images.

tom were measured by placing a circular ROI in the center of the aneurysms on raw data images. The ROI area was kept to $18 \mathrm{~mm}^{2}$. Because it is impossible to set the $18-\mathrm{mm}^{2}$ ROI on the $3-\mathrm{mm}$ aneurysms, we evaluated only the 6-mm aneurysms. In addition, the CT numbers of the background (silicone rubber) were measured by using the same ROI cursor. The CNR was calculated as follows: (ROIa $\mathrm{ROIb}$ ) / SDb, where ROIa and ROIb are the CT numbers of the artery ROI and background ROI, and SDb (background noise) is the SD of the CT numbers of the background.

Two neuroradiologists evaluated the image quality of the CTA by consensus according to the depiction of 6 simulated aneurysms with a diameter of $3 \mathrm{~mm}$ and 6 blebs by using a 5 -point score: $5=$ excellent (an aneurysm or aneurysmal bleb was depicted with same quality, which was close to that at the schematic drawing), $4=$ more than adequate (an aneurysm or aneurysmal bleb was clearly depicted, but image quality was somewhat reduced in comparison with that at the schematic drawing), 3 = adequate (depiction of the aneurysm or aneurysmal bleb was still sufficient), 2 = insufficient visualization, $1=$ not visible.

\section{Results}

Among the 6 combinations of tube voltages and effective tube currents with lower CTDIw than the standard CTA $(\mathrm{CTDIw}=48.0 \mathrm{mGy})$, the CTA with Q08 at $100 \mathrm{kVp}$ and 250 $\mathrm{mAs}(\mathrm{CTDIw}=39.8 \mathrm{mGy})$ and the CTA with Q08 at $120 \mathrm{kVp}$ and $150 \mathrm{mAs}$ (CTDIw $=36.0 \mathrm{mGy}$ ) showed significantly better results for both $\mathrm{CNR}$ and mean qualitative image score at 

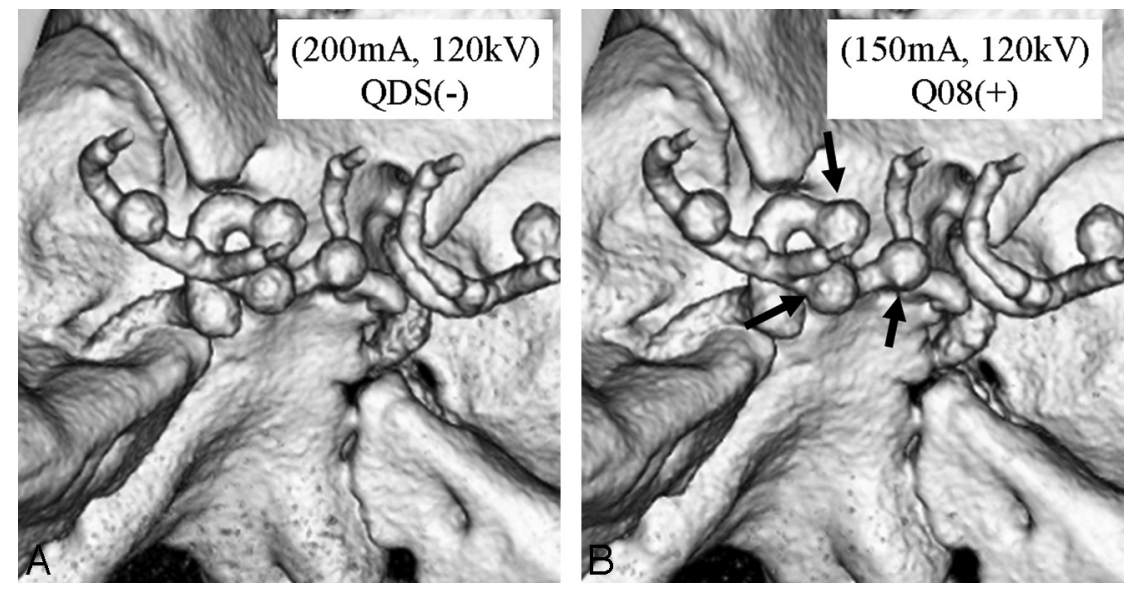

Fig 4. CTA without QDS with $120 \mathrm{kVp}$ and $200 \mathrm{mAs}(A)$ and Q08-processed CTA with $120 \mathrm{kVp}$ and $150 \mathrm{mAs}(B)$ from the superior view. For depiction of aneurysmal blebs on CTA at a concentration of $20 \mathrm{mg} \mathrm{l} / \mathrm{mL}$, the 008-processed CTA at $120 \mathrm{kVp}$ and $150 \mathrm{mAs}$ (arrows) is superior to the CTA without QDS at $120 \mathrm{kVp}$ and $200 \mathrm{mAs}$.

both concentrations of contrast material in comparison with the standard CTA without QDS (Fig 4), except for the qualitative imaging score with Q08 at $120 \mathrm{kVp}$ and $150 \mathrm{mAs}$ at 15 $\mathrm{mg} \mathrm{I} / \mathrm{mL}$ (Table).

\section{Discussion}

The current quantitative analysis with Wiener noise power spectra demonstrated that the QDS successfully reduced image noise on CT images, and the MTF of the filtered images was not substantially altered from that of the original image. These results indicate that the resolution of structured objects was preserved while noise reduction in nonstructured regions was achieved. The primary aim of this study was to determine how the radiation dose can be reduced by the use of QDS without compromising the image quality of the CTA. Kalra et $\mathrm{al}^{2}$ evaluated the adaptive filter designed to improve the quality of abdominal CT images obtained at a reduced tube current. Our pooled data at $120 \mathrm{kV}$ from the contrast material concentration of $20 \mathrm{mg} \mathrm{I} / \mathrm{mL}$ were analyzed and showed that the CTAs at $150 \mathrm{mAs}$ postprocessed with Q08 were significantly superior to the standard CTA at $200 \mathrm{mAs}$ with regard to the CNR and qualitative image score. On the basis of our analysis at $120 \mathrm{kV}$, we concluded that the scanning postprocessed with Q08 permits a reduction of the radiation dose of at least
25\% in comparison with the original images (CTDIw: 36.0 $\mathrm{mGy}$ at $150 \mathrm{mAs}$ versus $48.0 \mathrm{mGy}$ at $200 \mathrm{mAs}$ ).

There are some limitations in this study. In the 5-point analysis for the depiction of aneurysms, we used only the 3-mm aneurysms and blebs, because the 6-mm aneurysms could be detected too easily, which did not make any differences in the depiction among all the image protocols evaluated.

In conclusion, this phantom study demonstrated that the QDS reduced image noise without a perceptible loss of the delineation of structures of intracranial lesions and made it possible to reduce the radiation dose by $25 \%$ in intracranial CTA, while preserving the image quality or even allowing better image quality for the depiction of intracranial aneurysms and aneurysmal blebs.

\section{References}

1. Waaijer A, Prokop M, Velthuis BK, et al. Circle of Willis at CT angiography: dose reduction and image quality—reducing tube voltage and increasing tube current settings. Radiology 2007;242:832-39

2. Kalra MK, Maher MM, Sahani DV, et al. Low-dose CT of the abdomen: evaluation of image improvement with use of noise reduction filters pilot study. Radiology 2003;228:251-56. Epub 2003 May 8

3. Okumura M, Ota T, Kainuma K, et al. Effect of edge-preserving adaptive image filter on low-contrast detectability in CT systems: application of ROC analysis. Int J Biomed Imaging 2008:379-86. Epub 2008 Nov 16 